\title{
The "1-year-death number needed to treat" for comparing the impact of distinct interventions on patient outcomes
}

\author{
Brett N. Hryciw MD, Finlay A. McAlister MD MSc, Meltem Tuna MSc PhD, Carl van Walraven MD MSc
}

Cite as: CMAJ 2019 November 11;191:E1242-9. doi: 10.1503/cmaj.190367

A fter concluding that a randomized controlled trial (RCT) is both internally and externally valid, consumers of evidence may want to consider an intervention's overall importance compared with other interventions or health technologies. Such an exercise contributes to our perception of an intervention's importance to health. This process could also help health care organizations determine which of an assortment of new technologies should be introduced first, or identify processes of care that should be subjected to quality-of-care reviews and optimization. Finally, it might also help with treatment selection for an individual patient; for example, in a patient with newly diagnosed left ventricular systolic dysfunction, should one start treatment with angiotensin-converting-enzyme (ACE) inhibition or $\beta$-blockade?

However, making comparisons across trials and between different technologies can be difficult because studies differ with respect to patient populations, comparators (placebo v. active control interventions or active controls of differing efficacy), concomitant treatments, follow-up times and outcomes. Comparisons across trials from different eras are even more difficult given secular trends in health outcomes over time. Each of these factors can influence the baseline absolute risk of an event and, therefore, interstudy comparisons.

To help with this, we propose here a method to compare the impact of diverse interventions applied to different populations over varying observation times and with distinct outcomes using a summary statistic we call the 1-year-death number needed to treat (NNT). We illustrate the application of this method using a convenience sample of landmark RCTs. We then present the studied interventions in a league table of 1-year-death NNTs to show how the statistic can be used to gauge the impact of interventions on patient outcomes.

\section{What is the 1-year-death NNT?}

Although reported outcomes vary extensively between studies, all-cause mortality is commonly reported and is an outcome whose clinical importance is arguably independent of both the patient population and treatment type. We therefore used allcause mortality as the basis for the 1-year-death NNT. This statis-

\section{KEY POINTS}

- Cross-study comparisons of health care interventions are difficult because of varying patient populations, comparators, concomitant treatments and outcomes, which all affect the absolute risk of events.

- The 1-year-death number needed to treat (NNT) standardizes comparisons of treatment impact by focusing on all-cause mortality 1 year from treatment initiation using NNT methodology to account for distinct baseline risks.

- It is interpreted as the annual number of people requiring treatment and observation to avoid 1 death, with most beneficial treatments having positive values closest to 0 and most harmful treatments having negative values closest to 0 .

- The 1-year-death NNT permits the standardized comparison of diverse health care technologies, which could allow physicians to prioritize distinct treatment options for an individual patient or health care organizations to determine which new technologies to introduce first.

tic summarizes the influence of treatment on patient survival by accounting for between-study variations in baseline death risk and follow-up duration. It is calculated as

1-year death NNT = outcome time/ARR

in which ARR is the absolute risk reduction (calculated as death

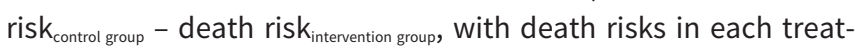
ment arm expressed as proportions from 0 to 1 and calculated as the number dying divided by the number randomly assigned). Outcome time is the number of years after randomization when death status was measured in the study. If the study did not follow all patients for the same amount of time, the outcome time is the average observation time. Some interventions have a finite treatment duration, with death status measured later on; in such cases, outcome time occurs at the end of the "treatment cycle," which includes both treatment time and the subsequent lag time before measurement of death status.

The 1-year-death NNT can have values that range from negative to positive infinity. The most beneficial treatments will have 


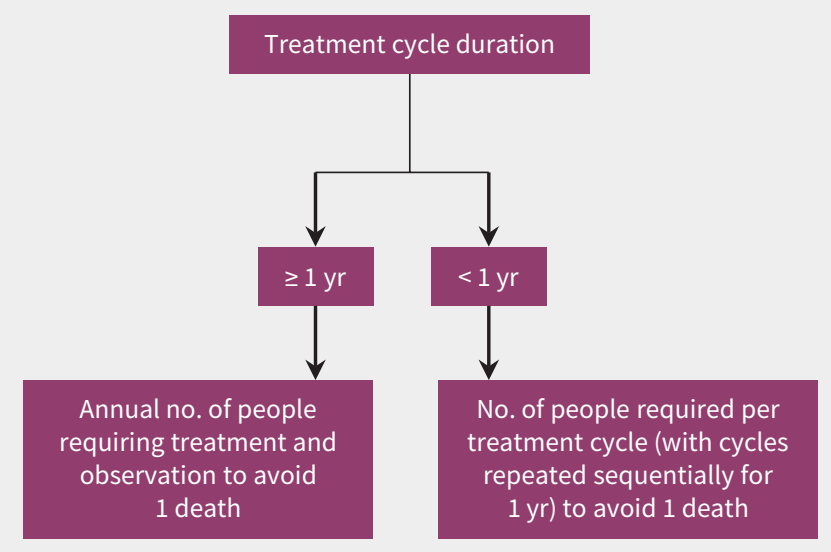

Figure 1: Interpretation of the 1-year-death number needed to treat. Note: treatment cycle duration = time from start of treatment to measurement of death status.

positive values closest to zero. Negative 1-year-death NNT values indicate interventions that increase death risk (with the absolute value of the 1-year-death NNT being interpreted as the "number needed to treat to harm"). Interpretation of the 1-yeardeath NNT depends on the treatment cycle duration (Figure 1). If the treatment cycle is 1 year or longer, the 1-year-death NNT is the annual number of people requiring treatment and observation to avoid 1 death. If the treatment cycle is less than a year, the 1-year-death NNT is the number of people required per treatment cycle (with cycles repeated sequentially for 1 year) to avoid 1 death. For example, consider a 1-year-death NNT of 5. If the treatment cycle is 2 years, this means that 5 people need treatment and observation for 1 year to avoid a single annual death; if the treatment cycle is 6 months, this means that 5 people need treatment and observation for 6 months twice (for a total of 10 treated people or 5 patient-years) to avoid a single annual death.

\section{How can the 1-year-death NNT be applied?}

To illustrate application of the 1-year-death NNT, we used as our sampling frame all RCTs included in 2 Minute Medicine's The Classics in Medicine: Summaries of the Landmark Trials. ${ }^{1}$ This is a collation of 190 "landmark clinical studies," defined by the authors as those identified by academic clinicians in general internal medicine and its subspecialties as "practice-changing," with a focus on common medical conditions (Dr. Andrew Cheung, St. Joseph's Healthcare Hamilton, Hamilton, Ont.: personal communication, 2019). We limited our convenience sample to RCTs (since these provide the least biased evaluations of treatment effects) and those reporting all-cause mortality (regardless of whether it was the study's primary outcome). Further methods and results are presented in Appendix 1 (available at www.cmaj. ca/lookup/suppl/doi:10.1503/cmaj.190367/-/DC1).

Table 1 summarizes the 31 RCTs reporting a significant difference in all-cause death risk between treatment groups. These trials included patients with congestive heart failure $(n=8)$, coro- nary artery disease $(n=5)$, intensive care issues $(n=4)$, established (or at a high risk of attaining) cardiovascular disease ( $n=$ $3)$, infectious diseases $(n=3)$, cirrhosis and its complications $(n=3)$, arrhythmia $(n=2)$, and 1 each of diabetes, premature labour and cancer. Publication dates ranged from 1972 to 2013.

Five trials $(16.1 \%)$ involved a procedural intervention, 13 trials (41.9\%) involved continuous treatments (i.e., daily medication), and all other studies ( $n=13,41.9 \%$ ) involved finite treatment durations ranging from 6 hours to 3 months (Table 2). Studies randomly assigned a mean of 5912 patients (range 61 to 45852 ). The mean outcome time was 1.7 years after randomization (range $1 \mathrm{wk}$ to $7.6 \mathrm{yr}$ ). Patient loss to follow-up was minimal in all studies except one. ${ }^{10}$ Death risks in control groups varied extensively between studies (range $2.8 \%$ to $88.5 \%$, median $22.7 \%$, mean $26.7 \%$ ). A total of 27 studies reported on interventions that decreased death risk, and the remaining 4 identified interventions that increased death risk.

The median 1-year-death NNT (in absolute values) was 18. The intervention saving the greatest number of lives per year (in this case, neonatal lives) was betamethasone during premature labour, with a 1-year-death NNT of 0.16 (95\% confidence interval [Cl] 0.09 to 0.64$).{ }^{2}$ Given this study's treatment cycle duration of less than 1 year, this means (Figure 1 ) that 0.16 mothers in premature labour required 1 day of betamethasone every week for a year (for a total of $0.16 \times 52=8.3$ people treated and observed) to avoid 1 death. Eleven interventions were beneficial and associated with a 1-year-death NNT of less than 10 (betamethasone in premature labour, prone ventilation in severe adult respiratory distress syndrome, prednisolone in alcoholic hepatitis, goaldirected resuscitation in sepsis, albumin in spontaneous bacterial peritonitis, voriconazole instead of amphotericin B in immunocompromised invasive aspergillosis, dexamethasone in acute meningitis, hypothermia after resuscitation, transjugular intrahepatic portosystemic shunting in acute variceal bleeding, tranexamic acid in trauma, and enalapril in severe congestive heart failure). ${ }^{2-12}$ The largest 1-year-death NNT (for prolonged tamoxifen after initial 5 years of treatment in early breast cancer) was $556(95 \% \mathrm{Cl} 272$ to $\infty)$.

Four trials $(12.9 \%)$ found a significantly increased death risk with the study's intervention (including strict glucose control for critical care patients [1-year-death NNT $-9.4,95 \% \mathrm{Cl}-63.8$ to $-5.1],{ }^{13}$ encainide or flecainide for frequent premature ventricular contractions after myocardial infarction [1-year-death NNT -21, $95 \% \mathrm{Cl}-47$ to -13$],{ }^{18}$ warfarin for symptomatic intracranial arterial stenosis [1-year-death NNT $-34,95 \% \mathrm{Cl}-148$ to -19$],{ }^{21}$ and intensive glucose control in high-risk patients with type 2 diabetes [1-year-death NNT $-334,95 \% \mathrm{Cl}-\infty$ to -54$\left.]^{30}\right)$.

\section{What can we learn from estimating the 1-year- death NNT?}

By calculating the 1-year-death NNT, we were able to standardize both changes in death risk between treatment groups and the time after randomization when death risk was calculated. This let us directly compare the impact of very distinct health care technologies on survival (Table 2). These benefits ranged from a life 
Table 1 (part 1 of 2): Summary of landmark randomized controlled trials in which treatments significantly reduced all-cause mortality

\begin{tabular}{|c|c|c|c|c|c|c|c|c|}
\hline Study & $\begin{array}{l}\text { Cochrane } \\
\text { bias risk* }\end{array}$ & $\begin{array}{l}\text { Run } \\
\text { int }\end{array}$ & $\begin{array}{l}\text { Multi- } \\
\text { centre‡ }\end{array}$ & $\begin{array}{l}\text { Industry } \\
\text { funded§ }\end{array}$ & Inclusion criteria & Interventionף & $\begin{array}{l}\text { Treatment } \\
\text { duration }\end{array}$ & Control** \\
\hline $\begin{array}{l}\text { Liggins and } \\
\text { Howie }^{2}\end{array}$ & L & $\mathrm{N}$ & N & Y & $\begin{array}{l}\text { Premature labour } \\
24-36 \text { wk }\end{array}$ & $\begin{array}{l}\text { Betamethasone } \\
\text { phosphate plus } \\
\text { betamethasone } \\
\text { acetate } 6 \mathrm{mg}\end{array}$ & $1 \mathrm{~d}$ & $\begin{array}{l}\text { Cortisone } \\
\text { acetate } 6 \text { mg }\end{array}$ \\
\hline Guérin et al. ${ }^{3}$ & U2 & $\mathrm{N}$ & Y & $\mathrm{N}$ & $\begin{array}{l}\text { Severe ARDS with ET } \\
\text { for }<36 \mathrm{~h}\end{array}$ & $\begin{array}{l}\text { Prone ventilation } \\
>16 \mathrm{~h} / \mathrm{d}\end{array}$ & $4 d$ & $\begin{array}{l}\text { Supine } \\
\text { ventilation }\end{array}$ \\
\hline $\begin{array}{l}\text { Ramond } \\
\text { et al. }{ }^{4}\end{array}$ & L & $\mathrm{N}$ & Y & $\mathrm{N}$ & $\begin{array}{l}\text { Alcoholic hepatitis on } \\
\text { biopsy with DF }>32 \text { or } \\
\text { HE }\end{array}$ & $\begin{array}{l}\text { Prednisolone } \\
40 \mathrm{mg} / \mathrm{d} \text { for } 28 \mathrm{~d}\end{array}$ & $28 d$ & Placebo \\
\hline Rivers et al. ${ }^{5}$ & L & $\mathrm{N}$ & $\mathrm{N}$ & Y & $\begin{array}{l}\text { Age } \geq 18 \text { yr, severe } \\
\text { sepsis, septic shock or } \\
\text { sepsis syndrome }\end{array}$ & $\begin{array}{l}\text { Goal-directed } \\
\text { resuscitation for } \\
6 \mathrm{~h}\end{array}$ & $6 \mathrm{~h}$ & None \\
\hline $\begin{array}{l}\text { de Gans and } \\
\text { van de Beek }\end{array}$ & L & $\mathrm{N}$ & Y & Y & Suspected meningitis & $\begin{array}{l}\text { Dexamethasone } \\
10 \mathrm{mg} \text { IV every } 6 \mathrm{~h}\end{array}$ & $4 d$ & Placebo \\
\hline $\begin{array}{l}\text { Hypothermia } \\
\text { after Cardiac } \\
\text { Arrest Study } \\
\text { Group }^{9}\end{array}$ & U3 & $\mathrm{N}$ & Y & Y & $\begin{array}{l}\text { Witnessed cardiac } \\
\text { arrest from VF or VT, } \\
\text { ROSC within } 60 \text { min }\end{array}$ & $\begin{array}{l}\text { Hypothermia } \\
32-34^{\circ} \mathrm{C} \text { for } 1 \mathrm{~d}\end{array}$ & $1 \mathrm{~d}$ & Normothermia \\
\hline $\begin{array}{l}\text { García-Pagán } \\
\text { et al. } .^{10}\end{array}$ & $H(2,3)$ & $\mathrm{N}$ & Y & $\mathrm{N}$ & $\begin{array}{l}\text { Cirrhosis, acute } \\
\text { variceal bleeding, } \\
\text { treatment with EGD } \\
\text { and VA drugs }\end{array}$ & TIPS within $72 \mathrm{~h}$ & - & $\begin{array}{l}\text { Endoscopic } \\
\text { treatment }\end{array}$ \\
\hline Finfer et al. ${ }^{13}$ & U3 & $\mathrm{N}$ & Y & $\mathrm{N}$ & $\begin{array}{l}\text { Admitted to ICU for } \\
\text { anticipated }>3 \mathrm{~d}\end{array}$ & $\begin{array}{l}\text { Target glucose } 4.5 \\
\text { to } 6.0 \mathrm{mmol} / \mathrm{L}\end{array}$ & $4.2 \mathrm{~d}$ & $\begin{array}{c}\text { Target glucose } \\
\leq 10 \mathrm{mmol} / \mathrm{L}\end{array}$ \\
\hline Rose et al. ${ }^{14}$ & U3 & $\mathrm{N}$ & Y & Y & $\begin{array}{l}\text { CHF, NYHA 4, low peak } \\
\text { oxygen consumption } \\
\text { and a contraindication } \\
\text { to transplantation }\end{array}$ & $\begin{array}{l}\text { Left ventricular } \\
\text { assist device }\end{array}$ & - & None \\
\hline $\begin{array}{l}\text { Malmberg } \\
\text { et al. }{ }^{15}\end{array}$ & $H(1,2)$ & $\mathrm{N}$ & Y & $\mathrm{N}$ & $\begin{array}{l}\text { Acute } \mathrm{MI} \text {, treated } \\
\text { diabetes or random } \\
\text { glucose }>11.1 \mathrm{mmol} / \mathrm{L}\end{array}$ & $\begin{array}{l}\text { IV insulin every } \\
24 \mathrm{~h} \text {, then } \\
\text { subsequently } \\
4 \text { times/d }\end{array}$ & $\geq 3 \mathrm{~min}$ & $\begin{array}{l}\text { "Conventional } \\
\text { therapy" }\end{array}$ \\
\hline Chen et al. ${ }^{16}$ & L & $N$ & Y & Y & $\begin{array}{l}\text { ST changes or LBBB } \\
\text { within } 24 \mathrm{~h} \text { of } \\
\text { symptoms }\end{array}$ & $\begin{array}{l}\text { Clopidogrel } \\
75 \mathrm{mg} / \mathrm{d} \text { plus ASA } \\
162 \mathrm{mg} / \mathrm{d}\end{array}$ & $15 d$ & ASA $162 \mathrm{mg} / \mathrm{d}$ \\
\hline Pitt et al. ${ }^{17}$ & L & $\mathrm{N}$ & Y & Y & LVEF $<35$, NYHA 3-4 & $\begin{array}{l}\text { Spironolactone } \\
25-50 \mathrm{mg} / \mathrm{d}\end{array}$ & $\infty$ & Placebo \\
\hline Echt et al. ${ }^{18}$ & U1 & Y & Y & $\mathrm{N}$ & $\begin{array}{l}\text { MI last } 2 \text { years, } \\
\geq 6 \mathrm{PVC} / \mathrm{h}, \mathrm{LVEF}<55 \% \text {, } \\
\text { response to treatment }\end{array}$ & $\begin{array}{l}\text { Encainide or } \\
\text { flecainide }\end{array}$ & $\infty$ & Placebo \\
\hline $\begin{array}{l}\text { MERIT-HF } \\
\text { Study Group }{ }^{19}\end{array}$ & L & Y & Y & Y & $\begin{array}{l}\text { CHF and NYHA } \geq 2, \\
\text { LVEF }<40 \%\end{array}$ & $\begin{array}{l}\text { Metoprolol XL } \\
12.5-200 \mathrm{mg} / \mathrm{d}\end{array}$ & $\infty$ & Placebo \\
\hline
\end{tabular}


Table 1 (part 2 of 2): Summary of landmark randomized controlled trials in which treatments significantly reduced all-cause mortality

\begin{tabular}{|c|c|c|c|c|c|c|c|c|}
\hline Study & $\begin{array}{l}\text { Cochrane } \\
\text { bias risk* }\end{array}$ & $\begin{array}{l}\text { Run } \\
\text { int }\end{array}$ & $\begin{array}{l}\text { Multi- } \\
\text { centreł }\end{array}$ & $\begin{array}{l}\text { Industry } \\
\text { funded§ }\end{array}$ & Inclusion criteria & Interventionף & $\begin{array}{l}\text { Treatment } \\
\text { duration }\end{array}$ & Control $^{\star \star}$ \\
\hline Moss et al..$^{20}$ & U2 & N & $Y$ & N & Prior MI, LVEF $<30$ & $I C D$ & - & None \\
\hline $\begin{array}{l}\text { Chimowitz } \\
\text { et al. }{ }^{21}\end{array}$ & L & N & $Y$ & Y & $\begin{array}{l}\text { Age } \geq 40 \mathrm{yr}, \text { TIA or } \\
\text { stroke from } 50 \%-99 \% \\
\text { ICA stenosis, Rankin } \\
\text { score } \leq 3\end{array}$ & Warfarin INR 2-3 & $\infty$ & ASA $1300 \mathrm{mg} / \mathrm{d}$ \\
\hline Bardy et al. ${ }^{22}$ & U2 & N & Y & Y & $\begin{array}{l}\text { Age } \geq 18 \text { yr, NYHA } 2-3 \\
\text { CHF, LVEF }<35 \%\end{array}$ & $\begin{array}{l}\text { Shock only, } \\
\text { single-lead ICD }\end{array}$ & - & None \\
\hline Yusuf et al. ${ }^{23}$ & L & Y & $Y$ & Y & $\mathrm{CHF}, \mathrm{LVEF}<35 \%$ & $\begin{array}{l}\text { Enalapril } \\
2.5-10 \mathrm{mg} \text { twice } \\
\text { daily }\end{array}$ & $\infty$ & Placebo \\
\hline $\begin{array}{l}\beta \text {-Blocker } \\
\text { Heart Attack } \\
\text { Trial }^{24}\end{array}$ & L & $\mathrm{N}$ & Y & Y & Age $30-69 \mathrm{yr}$, acute MI & $\begin{array}{l}\text { Propranolol } \\
40-80 \mathrm{mg} \\
3 \text { times/d }\end{array}$ & $\infty$ & Placebo \\
\hline Pfeffer et al..$^{25}$ & L & Y & $Y$ & Y & $\begin{array}{l}\text { Age } 21-80 \text { yr, LVEF } \\
<40 \% \text {, 3-16 d post-MI }\end{array}$ & $\begin{array}{l}\text { Captopril } \\
6.25-25 \mathrm{mg} \\
3 \text { times } / \mathrm{d}\end{array}$ & $\infty$ & Placebo \\
\hline $\begin{array}{l}\text { Wallentin } \\
\text { et al. }{ }^{26}\end{array}$ & $\mathrm{~L}$ & $\mathrm{~N}$ & Y & Y & $\begin{array}{l}\text { ACS presenting within } \\
24-\mathrm{h} \text { onset }\end{array}$ & $\begin{array}{l}\text { Ticagrelor } 90 \mathrm{mg} \\
\text { twice daily }\end{array}$ & $\infty$ & $\begin{array}{l}\text { Clopidogrel } \\
75 \mathrm{mg} / \mathrm{d}\end{array}$ \\
\hline $\begin{array}{l}\text { Farkouh } \\
\text { et al. }{ }^{27}\end{array}$ & $H(2,3)$ & N & Y & Y & $\begin{array}{l}\text { Diabetes, }>70 \% \\
\text { stenosis in } \geq 2 \text { major } \\
\text { epicardial vessels }\end{array}$ & CABG & - & $\mathrm{PCl}$ \\
\hline $\begin{array}{l}\text { Granger } \\
\text { et al. }{ }^{28}\end{array}$ & L & N & $Y$ & $\mathrm{Y}$ & $\begin{array}{l}\text { (Atrial fibrillation or } \\
\text { atrial flutter) + (age } \\
\geq 75 \text { yr or previous } \\
\text { stroke or TIA or } \\
\text { systemic embolism or } \\
\text { CHF or diabetes or } \\
\text { hypertension) }\end{array}$ & $\begin{array}{l}\text { Apixaban } 5 \mathrm{mg} \\
\text { twice daily }\end{array}$ & $\infty$ & Warfarin INR 2-3 \\
\hline Yusuf et al. ${ }^{29}$ & L & Y & Y & Y & $\begin{array}{l}\text { Age }>55 \text { yr, CAD, } \\
\text { stroke, PVD or } \\
\text { diabetes, and } \\
\text { (hypertension, } \\
\text { increased total } \\
\text { cholesterol, low HDL, } \\
\text { cigarette smoking or } \\
\text { microalbuminuria) }\end{array}$ & Ramipril 10 mg/d & $\infty$ & Placebo \\
\hline $\begin{array}{l}\text { Gerstein } \\
\text { et al. }{ }^{30}\end{array}$ & U2 & $\mathrm{N}$ & $\mathrm{Y}$ & $\mathrm{Y}$ & $\begin{array}{l}\text { Age } 40-79 \text { yr, type } 2 \\
\text { diabetes and } \mathrm{HbA}_{1 \mathrm{c}} \\
>7.5 \% \text {, high risk }\end{array}$ & $\begin{array}{l}\text { Intensive } \\
\text { treatment } \\
\text { targeting } \mathrm{HbA}_{1 \mathrm{c}} \\
<6 \%\end{array}$ & $\infty$ & $\begin{array}{c}\text { Treatment } \\
\text { targeting } \mathrm{HbA}_{1 \mathrm{c}} \\
7 \%-7.9 \%\end{array}$ \\
\hline Ridker et al. ${ }^{31}$ & U3 & $Y$ & $Y$ & $Y$ & $\begin{array}{l}\mathrm{LDL}<3.4, \mathrm{HS}-\mathrm{CRP} \\
>2 \mathrm{mg} / \mathrm{L}\end{array}$ & $\begin{array}{l}\text { Rosuvastatin } \\
20 \mathrm{mg} / \mathrm{d}\end{array}$ & $\infty$ & Placebo \\
\hline Davies et al. ${ }^{32}$ & U2 & N & $Y$ & $Y$ & $\begin{array}{l}\text { Early breast cancer, } \\
\text { tamoxifen } 5 \mathrm{yr}, \\
\text { disease-free }\end{array}$ & Tamoxifen & $5 \mathrm{yr}$ & None \\
\hline
\end{tabular}

Note: $\mathrm{ACS}=$ acute coronary syndrome, $\mathrm{ARDS}=$ adult respiratory distress syndrome, $\mathrm{ASA}=$ acetylsalicylic acid, $\mathrm{CABG}=$ coronary artery bypass graft, $\mathrm{CAD}=$ coronary artery disease, $\mathrm{CHF}=$ congestive heart failure, $D F=$ discriminant function (an algorithm to risk stratify severity of alcoholic hepatitis), $E G D=$ esophagogastroduodenoscopy, $E T=$ endotracheal tube, $H D L=$ high-density lipoprotein, $\mathrm{HE}=$ hepatic encephalopathy, $\mathrm{HbA}_{\mathrm{IC}}=$ glycated hemoglobin, $\mathrm{HS}$-CRP = high-sensitivity C-reactive protein, ICA = intracranial artery, ICD = implantable cardioverter-defibrillator, ICU = intensive care unit, INR = international normalized ratio, IV = intravenously, LBBB = left bundle branch block, LDL = low-density lipoprotein, LVEF = left ventricular ejection fraction, $\mathrm{MI}=$ myocardial infarction, $\mathrm{N}=$ no, NYHA = New York Heart Association Functional Classification, $P V D=$ peripheral vascular disease, $P C I=$ percutaneous coronary intervention, $P M N=$ polymorphonuclear cells, $P V C=$ premature ventricular contractions, $\mathrm{ROSC}=$ return of spontaneous circulation, $\mathrm{TIA}=$ transient ischemic attack, TIPS = transjugular intrahepatic portosystemic shunt, $\mathrm{VA}=$ vasoactive, $\mathrm{VF}=$ ventricular fibrillation, $\mathrm{VT}=$ ventricular tachycardia, $\mathrm{XL}=$ extended release, $\mathrm{Y}=\mathrm{yes},-=1$ procedure or surgery, $\infty=$ infinite or ongoing.

*Based on the Cochrane Collaboration's tool for assessing risk of bias in randomized trials ${ }^{33}$ focusing on domains most relevant to this study (allocation concealment, double-blinding and completeness of follow-up). $\mathrm{L}=$ low risk, $\mathrm{U}=$ uncertain risk, $\mathrm{H}=$ high risk. $1=$ no allocation concealment, $2=$ no double-blinding, $3=$ completeness of follow-up not cited or $<95 \%$. † $Y$ " if study had prerandomization observation time followed by criteria necessary for randomization.

" $Y$ " if patients were recruited at $>1$ study site.

$\S$ “" if study cited financial support from any company.

ITreatment for the intervention group.

${ }^{\star \star}$ Treatment for the control (or comparator) group. 
"being saved" annually after treatment of 8.3 mothers in premature labour with corticosteroids ${ }^{34}$ to continuing 556 women with early breast cancer on tamoxifen (after an initial 5 years of treatment) for another year. ${ }^{32}$ Table 2 shows that, even after limiting ourselves to the "best of the best" landmark studies (i.e., recognized landmark RCTs of interventions that significantly influenced all-cause mortality), large variations remained in the interventions' influence on patient survival. These results highlight that the critical review of RCTs should not stop with "significantly decreased all-cause mortality."

The 1-year-death NNT and Table 2 help put RCT outcomes into perspective and better gauge the importance of interventions on patient survival. However, the interventions presented in these 31 studies should all be considered special. All-cause mortality is critical to patients and is almost invariably measured accurately by researchers, making it a key RCT outcome. Interventions that significantly change the likelihood of death beyond that expected by chance alone are important. If desired, the formula presented above could be modified to calculate 1-year standardized NNTs for any outcome that one cares to compare between studies.

\section{What are the potential limitations of our example?}

Any method used to sample all published studies evaluating treatments has its drawbacks. We used a collection of studies subjectively identified by experts to be important to and influential on clinical practice. ${ }^{1}$ However, there is considerable overlap between studies included in The Classics ${ }^{1}$ and other collections of key studies; almost a third of the trials published between 1991 and 2003 in The Classics were included in loannidis' seminal report of landmark RCTs, ${ }^{35}$ and more than $40 \%$ of RCTs in Hochman's collection of key studies ${ }^{36}$ were in The Classics. ${ }^{1}$ Our study may have excluded key RCTs reporting important treatment effects on all-cause survival. Further work is required to systematically identify all other methodologically robust RCTs that report a significant influence of treatment on all-cause survival and adding them to our table.

Two issues arise from our results being based on RCT outcomes. First, with the exception of ACE inhibitors ${ }^{12,23,25}$ and implantable cardioverter-defibrillators ${ }^{20,22}$ for left ventricular systolic dysfunction, results in our league table are based on a single

Table 2 (part 1 of 2): All-cause mortality outcomes in landmark randomized controlled trials with statistically significant results

\begin{tabular}{|c|c|c|c|c|c|c|c|c|c|c|}
\hline \multirow[b]{2}{*}{ Study } & \multirow{2}{*}{$\begin{array}{l}\text { Treatment, } \\
\text { no.* }^{*}\end{array}$} & \multirow{2}{*}{$\begin{array}{c}\text { Control, } \\
\text { no. }\end{array}$} & \multirow{2}{*}{$\begin{array}{l}\text { Treatment } \\
\text { duration } \neq\end{array}$} & \multirow{2}{*}{$\begin{array}{l}\text { Outcome } \\
\text { time, yr§ }\end{array}$} & \multicolumn{2}{|c|}{ Death riskף } & \multirow[b]{2}{*}{ ARR‡¥ } & \multirow{2}{*}{$\begin{array}{c}p \\
\text { value§§ }\end{array}$} & \multirow{2}{*}{$\begin{array}{c}\text { NNT- } \\
\text { outcome } \\
\text { timeฯฯ }\end{array}$} & \multirow{2}{*}{$\begin{array}{l}\text { 1-year-death } \\
\text { NNT }(95 \% \mathrm{Cl})^{\star \star \star}\end{array}$} \\
\hline & & & & & Treatment ${ }^{\star \star}$ & Controltt & & & & \\
\hline Guérin et al. ${ }^{3}$ & 237 & 229 & $4 d$ & 0.0767 & 0.236 & 0.41 & 0.174 & $<0.001$ & 5.7 & 0.4 (1.0 to 2.7 ) \\
\hline Sort et al. ${ }^{6}$ & 63 & 63 & $3 d$ & 0.2466 & 0.222 & 0.413 & 0.19 & 0.03 & 5.3 & $1.3(0.7$ to 7.9$)$ \\
\hline $\begin{array}{l}\text { Herbrecht } \\
\text { et al. }{ }^{7}\end{array}$ & 144 & 133 & $12 \mathrm{wk}$ & 0.2308 & 0.292 & 0.414 & 0.122 & 0.02 & 8.2 & 1.9 (1.0 to 23 ) \\
\hline $\begin{array}{l}\text { de Gans and } \\
\text { van de Beek }\end{array}$ & 157 & 144 & $4 d$ & 0.1538 & 0.07 & 0.146 & 0.076 & 0.04 & 13.2 & 2.0 (1.1 to 27$)$ \\
\hline $\begin{array}{l}\text { García-Pagán } \\
\text { et al. }{ }^{10}\end{array}$ & 32 & 31 & - & 1.3333 & 0.125 & 0.387 & 0.262 & 0.01 & 3.8 & 5.1 (2.1 to 18$)$ \\
\hline $\begin{array}{l}\text { Williams- } \\
\text { Johnson et al. }{ }^{11}\end{array}$ & 10060 & 10067 & $8 \mathrm{~h}$ & 0.0769 & 0.145 & 0.16 & 0.015 & 0.0035 & 67.6 & 5.2 (3.1 to 16$)$ \\
\hline $\begin{array}{l}\text { CONSENSUS } \\
\text { Trial Study } \\
\text { Group }^{12}\end{array}$ & 127 & 126 & $\infty$ & 0.9863 & 0.362 & 0.524 & 0.162 & 0.003 & 6.2 & 6.1 (3.5 to 24$)$ \\
\hline Finfer et al. ${ }^{13}$ & 3010 & 3012 & $4.2 \mathrm{~d}$ & 0.2466 & 0.275 & 0.249 & -0.026 & 0.02 & -38.3 & $-9.5(-64$ to -5.1$)$ \\
\hline Rose et al. ${ }^{14}$ & 61 & 61 & - & 2.1667 & 0.672 & 0.885 & 0.213 & $<0.001$ & 4.7 & 10 (2.8 to 14$)$ \\
\hline
\end{tabular}


Table 2 (part 2 of 2): All-cause mortality outcomes in landmark randomized controlled trials with statistically significant results

\begin{tabular}{|c|c|c|c|c|c|c|c|c|c|c|}
\hline \multirow[b]{2}{*}{ Study } & \multirow{2}{*}{$\begin{array}{c}\text { Treatment, } \\
\text { no.* }\end{array}$} & \multirow{2}{*}{$\begin{array}{c}\text { Control, } \\
\text { no.† }\end{array}$} & \multirow{2}{*}{$\begin{array}{l}\text { Treatment } \\
\text { duration } \ddagger\end{array}$} & \multirow{2}{*}{$\begin{array}{l}\text { Outcome } \\
\text { time, yr§ }\end{array}$} & \multicolumn{2}{|c|}{ Death riskๆ } & \multirow[b]{2}{*}{ ARR‡¥ } & \multirow{2}{*}{$\stackrel{p}{p}$} & \multirow{2}{*}{$\begin{array}{c}\text { NNT- } \\
\text { outcome } \\
\text { timeๆף }\end{array}$} & \multirow{2}{*}{$\begin{array}{l}\text { 1-year-death } \\
\text { NNT }(95 \% \mathrm{CI})^{\star \star \star}\end{array}$} \\
\hline & & & & & Treatment $^{\star \star}$ & Controltt & & & & \\
\hline $\begin{array}{l}\text { MERIT-HF } \\
\text { Study Group }{ }^{19}\end{array}$ & 1990 & 2001 & $\infty$ & 1 & 0.073 & 0.108 & 0.036 & $<0.001$ & 28.1 & 28 (19 to 56$)$ \\
\hline Moss et al. ${ }^{20}$ & 742 & 490 & - & 1.6667 & 0.142 & 0.198 & 0.056 & 0.016 & 17.7 & 30 (17 to 127$)$ \\
\hline $\begin{array}{l}\text { Chimowitz } \\
\text { et al. }{ }^{21}\end{array}$ & 289 & 280 & $\infty$ & 1.85 & 0.097 & 0.043 & -0.054 & 0.02 & -18.5 & $-34(-148$ to -19$)$ \\
\hline Bardy et al. ${ }^{22}$ & 829 & 847 & - & 3.7917 & 0.22 & 0.288 & 0.069 & 0.007 & 14.6 & 55 (45 to 185 ) \\
\hline Yusuf et al..$^{23}$ & 1,285 & 1,284 & $\infty$ & 3.45 & 0.352 & 0.397 & 0.045 & 0.0036 & 22 & 76 (42 to 428 ) \\
\hline $\begin{array}{l}\text { B-Blocker } \\
\text { Heart Attack } \\
\text { Trial }^{24}\end{array}$ & 1916 & 1921 & $\infty$ & 2.0833 & 0.072 & 0.098 & 0.026 & $<0.005$ & 38.7 & 81 (69 to 365$)$ \\
\hline Pfeffer et al. ${ }^{25}$ & 1115 & 1116 & $\infty$ & 3.5 & 0.204 & 0.246 & 0.042 & 0.019 & 23.8 & 83 (46 to 480$)$ \\
\hline $\begin{array}{l}\text { Wallentin } \\
\text { et al. }{ }^{26}\end{array}$ & 9333 & 9291 & $\infty$ & 1 & 0.043 & 0.054 & 0.012 & $<0.001$ & 85.4 & 85 (56 to 181$)$ \\
\hline $\begin{array}{l}\text { Farkouh } \\
\text { et al. }{ }^{27}\end{array}$ & 947 & 953 & - & 5 & 0.088 & 0.12 & 0.032 & 0.049 & 31.3 & 156 (84 to 1084$)$ \\
\hline Yusuf et al. ${ }^{29}$ & 4645 & 4652 & $\infty$ & 3.5 & 0.104 & 0.122 & 0.019 & 0.005 & 53.9 & 270 (159 to 881$)$ \\
\hline $\begin{array}{l}\text { Gerstein } \\
\text { et al. }{ }^{30}\end{array}$ & 5128 & 5123 & $\infty$ & 1.9 & 0.05 & 0.04 & -0.01 & 0.04 & -95.3 & $-334(\infty$ to -54$)$ \\
\hline Ridker et al. ${ }^{31}$ & 8901 & 8901 & $\infty$ & 7.6 & 0.022 & 0.028 & 0.006 & 0.02 & 181.7 & $345(99$ to $\infty)$ \\
\hline Davies et al. ${ }^{32}$ & 6454 & 6440 & $5 \mathrm{yr}$ & 7.6 & 0.214 & 0.227 & 0.014 & 0.04 & 73.2 & $556(272$ to $\infty)$ \\
\hline 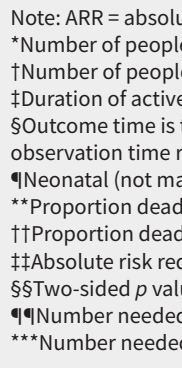 & $\begin{array}{l}\text { e risk reduction, } \\
\text { randomly assigne } \\
\text { randomly assigne } \\
\text { reatment for the } \\
\text { e time from rand } \\
\text { quired to achieve } \\
\text { ernal) death risk. } \\
\text { n intervention gro } \\
\text { n control group ( } \\
\text { iction for death, c } \\
\text { for comparison } \\
\text { o treat to preven } \\
\text { to treat to preven }\end{array}$ & $\begin{array}{l}\text { I confidence } \\
d \text { to interventi } \\
\text { d to control gr } \\
\text { ntervention } g \\
\text { mization at } w \\
\text { observed deat } \\
\text { up (no. dead } \\
\text { o. dead } \div \text { no. } \\
\text { lculated as d } \\
\text { f death risk in } \\
1 \text { death by ou } \\
1 \text { death by } 1 \text {. }\end{array}$ & $\begin{array}{l}\text { interval, NNT = n } \\
\text { n group. } \\
\text { oup. } \\
\text { oup. } \\
\text { hich death count } \\
\text { h risk. In open st } \\
\text { no. randomly as } \\
\text { andomly assigne } \\
\text { ath risk for contr } \\
\text { groups. } \\
\text { come time. } \\
\text { ear. If negative, t }\end{array}$ & $\begin{array}{l}\text { umber needed } \\
\text { or risk was med } \\
\text { dies (i.e., outcc } \\
\text { igned). } \\
\text { d). } \\
\text { l group - deat } \\
\text { is is the numb }\end{array}$ & $\begin{array}{l}\text { to treat, - = } 1 \text { proce } \\
\text { sured. In closed stu } \\
\text { me time varies bet }\end{array}$ & $\begin{array}{l}\text { lure or surgery, } \\
\text { dies, it equals th } \\
\text { veen patients in }\end{array}$ & $\begin{array}{l}\text { = ongoing } \\
\text { e time patie } \\
\text { study), the }\end{array}$ & $\begin{array}{l}\text { treatment thr } \\
\text { ents received a } \\
\text { average follow }\end{array}$ & $\begin{array}{l}\text { ughout obser } \\
\text { ctive treatmen } \\
\text {-up time was u }\end{array}$ & $\begin{array}{l}\text { vation. } \\
\text { t plus subsequent } \\
\text { sed. }\end{array}$ \\
\hline
\end{tabular}

RCT of interventions. Single studies, even those that are large and internally valid, can return exaggerated results that can be smaller (or sometimes even absent) when trials are replicated or when the intervention is applied in clinical practice. ${ }^{35}$ This issue could be addressed using data from meta-analyses rather than single studies. Second, the intervention effect measured in RCTs may generalize poorly. ${ }^{37}$

Other issues regarding our example should be kept in mind. First, some of the health care technologies compared in our study are quite distinct, making their direct comparison relevant only in a numerical sense regarding the 1-year-death NNT. Second, we reported risk using a simple proportion because this was reported in all studies. Risk-measurement methods that account for observation time will more accurately measure risk; such measurements can be used instead of proportions if calculable in the RCTs of all technologies that are to be compared. Finally, we used the $p$ value for the intertreatment difference in death risk to select treatments that were highlighted in Table 2. This dichotomization of a continuous measure (i.e., the likelihood that differences seen exceed that arising by chance) will unfairly exclude some studies (such as those with a $p$ value of 0.05 ) whose importance is not materially distinct from those presented here.

\section{What are the potential limitations of using the 1-year-death NNT statistic?}

Several potential limitations of the number needed to treat (NNT) will also apply to the 1 -year-death NNT. ${ }^{38,39}$ First, both statistics will tend to increase extensively when baseline risks decrease. ${ }^{38}$ Second, baseline death risks tend to exhibit secular decreases over time as concomitant therapies and other aspects of medical care improve; for example, 1-year mortality for adults admitted to hospital in Ontario decreased by $20 \%$ between 1994 and 2009. This makes comparisons of NNT and 
1-year-death NNT between years problematic. ${ }^{40}$ Third, death risk estimates - and differences between treatment groups - get larger the longer patients are observed. Therefore, the NNT (the reciprocal of the absolute risk reduction) will decrease as time from randomization to outcome measurement increases. ${ }^{38}$ The 1-year-death NNT avoids this limitation by standardizing all NNT time frames to 1 year after randomization. However, this standardization assumes that the relative influence of treatment on death risk is the same 1 year after randomization as at the study's reported outcome time. If treatment effect changes significantly over time, the 1-year-death NNT may return a biased estimate. The outcome times of almost half of the studies we analyzed $(n=15)$ were very close to 1 year (between 6 mo and $2 \mathrm{yr}$ ), thereby reducing the influence of this potential bias. Fourth, the 1-year-death NNT simplifies the comparison of absolute death risk with potentially important loss of information. Therefore, one should always examine the absolute death risks in each patient group to truly appreciate treatment effects. Fifth, the 1-year-death NNTs reported in Table 2 (just as with all NNT measures) represent the average value of patients in those trials; values could vary widely in particular subgroups. Finally, in RCTs having variable observation time for patients, death risks were most accurate when censoring is considered using survival analysis techniques. ${ }^{41}$ Since most RCTs in our worked example did not use survival analysis or provide the necessary data to permit recalculation, death risks were summarized using proportions. This made interstudy comparisons possible but could underestimate death risk in studies with long observation times and large treatment effects.

Several limitations are particular to the 1-year death NNT. First, in using all-cause mortality to permit cross-study and crosstechnology comparisons, we assumed that all deaths are equal. This is arguably not true since some deaths are symptomatically worse than others (e.g., contrast a person dying in their sleep to someone dying with progressive, incapacitating respiratory failure). Further research might explore the possibility of weighting deaths using health utilities and health-related quality of life measures. ${ }^{42}$ Second, the 1-year-death NNT also does not account for life years lost owing to the death. Neonatal death during premature labour is more consequential with regard to population health than death in an older adult. Further work could explore weighting 1-year-death NNT by expected life years lost. Third, interventions might provide important benefits to patients without influencing the likelihood of death. Fourth, 1-year-death NNT is but one statistic that could be used to compare health care technologies. Other factors, such as cost and disease prevalence, should also be considered. Finally, the 1-year-death NNT will vary with the outcome time chosen in the RCT. Consider a study in which deaths in both treatment groups are primarily clustered close to the start of observation; the corresponding survival curves will be increasingly flat over time. If such a study chose an observation time that notably exceeded the period during which deaths occurred, 1-year-death NNT would return an inappropriately large value. This possibility supports the point made above: to truly understand the 1-year-death NNT (as with any summary statistic), one should always examine its individual data components.

\section{Conclusion}

Despite its limitations, the 1-year-death NNT represents a useful statistic to help consumers of medical evidence contextualize potential impacts of health care interventions. It could also help health care organizations prioritize technologies for evaluation or funding and help individual physicians discriminate between distinct treatment options.

\section{References}

1. Succi MD, Carr LH, Cheung A. 2 Minute Medicine's the classics in medicine: summaries of the landmark trials. 1st ed. Boston: 2 Minute Medicine Inc.; 2015.

2. Liggins GC, Howie RN. A controlled trial of antepartum glucocorticoid treatment for prevention of the respiratory distress syndrome in premature infants. Pediatrics 1972;50:515-25.

3. Guérin C, Reignier J, Richard JC, et al.; PROSEVA Study Group. Prone positioning in severe acute respiratory distress syndrome. N Engl J Med 2013;368:2159-68.

4. Ramond MJ, Poynard T, Rueff B, et al. A randomized trial of prednisolone in patients with severe alcoholic hepatitis. N Engl J Med 1992;326:507-12.

5. Rivers E, Nguyen B, Havstad S, et al.; Early Goal-Directed Therapy Collaborative Group. Early goal-directed therapy in the treatment of severe sepsis and septic shock. N Engl J Med 2001;345:1368-77.

6. Sort P, Navasa M, Arroyo V, et al. Effect of intravenous albumin on renal impairment and mortality in patients with cirrhosis and spontaneous bacterial peritonitis. N Engl J Med 1999;341:403-9.

7. Herbrecht R, Denning DW, Patterson TF, et al.; Invasive Fungal Infections Group of the European Organisation for Research and Treatment of Cancer and the Global Aspergillus Study Group. Voriconazole versus amphotericin B for primary therapy of invasive aspergillosis. N Engl J Med 2002;347:408-15.

8. de Gans J, van de Beek D; European Dexamethasone in Adulthood Bacterial Meningitis Study Investigators. Dexamethasone in adults with bacterial meningitis. N Engl J Med 2002;347:1549-56.

9. Hypothermia after Cardiac Arrest Study Group. Mild therapeutic hypothermia to improve the neurologic outcome after cardiac arrest. N Engl J Med 2002;346: 549-56.

10. García-Pagán JC, Caca K, Bureau C, et al.; Early TIPS (Transjugular Intrahepatic Portosystemic Shunt) Cooperative Study Group. Early use of TIPS in patients with cirrhosis and variceal bleeding. N Engl J Med 2010;362:2370-9.

11. Williams-Johnson JA, McDonald AH, Strachan GG, et al. Effects of tranexamic acid on death, vascular occlusive events, and blood transfusion in trauma patients with significant haemorrhage (CRASH-2): a randomised, placebocontrolled trial. West Indian Med J 2010;59:612-24.

12. CONSENSUS Trial Study Group. Effects of enalapril on mortality in severe congestive heart failure. Results of the Cooperative North Scandinavian Enalapril Survival Study (CONSENSUS). N Engl J Med 1987;316:1429-35.

13. NICE-SUGAR Study Investigators; Finfer S, Chittock DR, Su SY, et al. Intensive versus conventional glucose control in critically ill patients. N Engl J Med 2009; 360:1283-97.

14. Rose EA, Gelijns AC, Moskowitz AJ, et al.; Randomized Evaluation of Mechanical Assistance for the Treatment of Congestive Heart Failure (REMATCH) Study Group. Long-term use of a left ventricular assist device for end-stage heart failure. N Engl J Med 2001;345:1435-43.

15. Malmberg K, Rydén L, Efendic S, et al. Randomized trial of insulin-glucose infusion followed by subcutaneous insulin treatment in diabetic patients with acute myocardial infarction (DIGAMI study): effects on mortality at 1 year. J Am Coll Cardiol 1995;26:57-65.

16. Chen ZM, Jiang LX, Chen YP, et al.; COMMIT (ClOpidogrel and Metoprolol in Myocardial Infarction Trial) collaborative group. Addition of clopidogrel to aspirin in 45,852 patients with acute myocardial infarction: randomised placebocontrolled trial. Lancet 2005;366:1607-21.

17. Pitt B, Zannad F, Remme WJ, et al. The effect of spironolactone on morbidity and mortality in patients with severe heart failure. N Engl J Med 1999;341:709-17.

18. Echt DS, Liebson PR, Mitchell LB, et al. Mortality and morbidity in patients receiving encainide, flecainide, or placebo. The Cardiac Arrhythmia Suppression Trial. N Engl J Med 1991;324:781-8.

19. Effect of metoprolol CR/XL in chronic heart failure: Metoprolol CR/XL Randomised Intervention Trial in Congestive Heart Failure (MERIT-HF). Lancet 1999;353:2001-7. 
20. Moss AJ, Zareba W, Hall WJ, et al.; Multicenter Automatic Defibrillator Implantation Trial II Investigators. Prophylactic implantation of a defibrillator in patients with myocardial infarction and reduced ejection fraction. N Engl J Med 2002;346:877-83.

21. Chimowitz MI, Lynn MJ, Howlett-Smith H, et al.; Warfarin-Aspirin Symptomatic Intracranial Disease Trial Investigators. Comparison of warfarin and aspirin for symptomatic intracranial arterial stenosis. N Engl J Med 2005;352:1305-16.

22. Bardy GH, Lee KL, Mark DB, et al. Amiodarone or an implantable cardioverterdefibrillator for congestive heart failure. N Engl J Med 2005;352:225-37.

23. SOLVD Investigators; Yusuf S, Pitt B, Davis CE, et al. Effect of enalapril on survival in patients with reduced left ventricular ejection fractions and congestive heart failure. N Engl J Med 1991;325:293-302.

24. A randomized trial of propranolol in patients with acute myocardial infarction: I. mortality results. JAMA 1982;247:1707-14.

25. Pfeffer MA, Braunwald E, Moyé LA, et al. Effect of captopril on mortality and morbidity in patients with left ventricular dysfunction after myocardial infarction. Results of the survival and ventricular enlargement trial. The SAVE Investigators. N Engl J Med 1992;327:669-77.

26. Wallentin L, Becker RC, Budaj A, et al. Ticagrelor versus clopidogrel in patients with acute coronary syndromes. N Engl J Med 2009;361:1045-57.

27. Farkouh ME, Domanski M, Sleeper LA, et al.; FREEDOM Trial Investigators. Strategies for multivessel revascularization in patients with diabetes. $N$ Engl J Med 2012;367:2375-84.

28. Granger CB, Alexander JH, McMurray JJV, et al.; ARISTOTLE Committees and Investigators. Apixaban versus warfarin in patients with atrial fibrillation. N Engl J Med 2011;365:981-92.

29. Heart Outcomes Prevention Evaluation Study Investigator; Yusuf S, Sleight $P$, Pogue J, et al. Effects of an angiotensin-converting enzyme inhibitor, ramipril, on cardiovascular events in high-risk patients [published erratum in N Engl J Med 2000;342:748, 1376]. N Engl J Med 2000;342:145-53.

30. Action to Control Cardiovascular Risk in Diabetes Study Group; Gerstein HC, Miller ME, Byington RP, et al. Effects of intensive glucose lowering in type 2 diabetes. N Engl J Med 2008;358:2545-59.
31. Ridker PM, Danielson E, Fonseca FAH, et al.; JUPITER Study Group. Rosuvastatin to prevent vascular events in men and women with elevated C-reactive protein. N Engl J Med 2008;359:2195-207.

32. Davies C, Pan H, Godwin J, et al.; Adjuvant Tamoxifen: Longer Against Shorter (ATLAS) Collaborative Group. Long-term effects of continuing adjuvant tamoxifen to 10 years versus stopping at 5 years after diagnosis of oestrogen receptorpositive breast cancer: ATLAS, a randomised trial. Lancet 2013;381:805-16.

33. Higgins JPT, Altman DG, Goetzsche PC, et al.; Cochrane Bias Methods Group; Cochrane Statistical Methods Group. The Cochrane Collaboration tool for assessing risk of bias in randomised trials. BMJ 2011;343:d5928.

34. Bardy GH, Lee KL, Mark DB, et al.; Sudden Cardiac Death in Heart Failure Trial (SCD-HeFT) Investigators. Amiodarone or an implantable cardioverter-defibrillator for congestive heart failure. N Engl J Med 2005;352:225-37.

35. Ioannidis JPA. Contradicted and initially stronger effects in highly cited clinical research. JAMA 2005;294:218-28.

36. Hochman ME. 50 studies every doctor should know: the key studies that form the foundation of evidence based medicine. Revised ed. Oxford (UK): Oxford University Press; 2013.

37. Bailey KR. Generalizing the results of randomized clinical trials. Control Clin Trials 1994;15:15-23.

38. McAlister FA. The "number needed to treat" turns $20-$ and continues to be used and misused. CMAJ 2008;179:549-53.

39. Suissa D, Brassard P, Smiechowski B, et al. Number needed to treat is incorrect without proper time-related considerations. J Clin Epidemiol 2012;65:42-6.

40. van Walraven C. Trends in 1-year survival of people admitted to hospital in Ontario, 1994-2009. CMAJ 2013;185:E755-62.

41. Zee J, Xie SX. The Kaplan-Meier method for estimating and comparing proportions in a randomized controlled trial with dropouts. Biostat Epidemiol 2018;2:23-33.

42. Horsman J, Furlong W, Feeny D, et al. The Health Utilities Index (HUI): concepts, measurement properties and applications. Health Qual Life Outcomes 2003;1:54.

\section{Competing interests: None declared.}

This article has been peer reviewed.

Affiliations: Department of Medicine (Hryciw, van Walraven), The Ottawa Hospital, Ottawa, Ont.; Division of General Internal Medicine (McAlister), University of Alberta, Edmonton, Alta.; ICES uOttawa (Tuna, van Walraven); Ottawa Hospital Research Institute (Tuna, van Walraven), Ottawa, Ont.
Contributors: Brett Hryciw and Carl van Walraven contributed to the conception and data collection. Brett Hryciw, Carl van Walraven and Meltem Tuna contributed to the data analysis. All of the authors contributed to the data interpretation. Carl van Walraven and Finlay McAlister drafted the article, which all of the authors reviewed. All of the authors gave final approval of the version to be published and agreed to be accountable for all aspects of the work.

Correspondence to: Carl van Walraven, carlv@ohri.ca 